\title{
Predictive Factors for Postrecurrence Survival in Epithelial Ovarian Cancer-a Consecutive Series of 368 Patients and Review of the Literature
}

Petru $E^{1 *}$, Idris $\mathrm{T}^{1}$, Woltsche $\mathrm{N}^{2}$, Haas J ${ }^{1}$, Benedicic C ${ }^{1}$, Heydar-Fadai $M^{1}$, Kurschel-Lackner $\mathrm{S}^{\mathbf{1}, 3}$, Walsberger $\mathrm{K}^{\mathbf{1}}$ and Tamussino $\mathrm{K}^{\mathbf{1}}$ ${ }^{1}$ Department of Obstetrics and Gynecology, Medical University of Graz, Austria

${ }^{2}$ Department of Ophthalmology, Medical University of Graz, Austria

${ }^{3}$ Department of Neurosurgery, Medical University of Graz, Austria

*Corresponding author: Edgar Petru, Department of Obstetrics and Gynecology, Division of Gynecology, Medical University of Graz, Auenbruggerplatz, Austria

Received: November 12, 2018; Accepted: December 27, 2018; Published: December 31, 2018

\begin{abstract}
Purpose: Ovarian cancer is usually diagnosed at advanced stage. Thus, recurrences are common. The aim of this retrospective study was to analyze the prognostic value of selected clinical and biological factors with regard to overal survival after first recurrence.

Methods: A total of 368 evaluable patients with primary epithelial cancer of the ovary, fallopian tube or peritoneum were included. Carcinosarcomas, sarcomas and borderline tumors of the ovary, the fallopian tube and the peritoneum were excluded. Patient and tumor characteristics were extracted from hospital records. Recurrence was defined as the first clinical manifestation of tumor progression after an interval of no clinical evidence of disease following primary surgery. Patients with an elevated tumor marker CA125 alone and those who had clinically evident tumor after primary surgery were not eligible for this study.
\end{abstract}

Results: In the multivariate cox regression analysis, five parameters were identified as independent favourable prognostic factors for survival after first recurrence: Time to recurrence $\geq 2$ years ( $p=0.000)$, Karnofsky status $\geq 80 \%$ at the time of recurrence $(p=0.008)$, use of adjuvant chemotherapy $(p=0.013)$, residual disease $\leq 1 \mathrm{~cm}$ at primary surgery $(p=0.044)$, and isolated peripheral or paraaortic lymph nodes as localization of first recurrence $(p<0.05)$.

Conclusions: Prolonged interval to recurrence seems to be of utmost importance for longer postrecurrence survival. In addition, small or no residual tumor after primary surgery, administration of adjuvant chemotherapy, higher performance status at recurrence and metastases in the peripheral or paraaortic nodes are predictive for improved overall survival.

Keywords: First recurrence; Ovarian cancer; Prognosis; Survival

\section{Introduction}

Most patients with ovarian cancer are diagnosed at an advanced stage, and most will develop recurrence [1]. The abdominal cavity and retroperitoneal lymph nodes are the most frequent sites of first recurrence [1] while distant metastases are uncommon [1,2]. There are inconsistent data on predictive factors for overall survival after first recurrence of epithelial ovarian cancer [3-12].

This retrospective study aimed to describe the patterns of first recurrence and to analyze the predictive value of available clinical and tumor-related factors in patients with ovarian cancer treated and followed up in a single academic institution.

\section{Methods}

Between 1976 and 2009, a total of 1,003 consecutive patients with primary epithelial cancer of the ovary, fallopian tube or the peritoneum were treated at the Division of Gynecology, Department of Obstetrics and Gynecology at the Medical University of Graz. Carcinosarcomas, primary sarcomas and borderline were excluded as were tumors metastatic to the ovary. Patient and tumor characteristics were extracted from hospital records and are listed in Table 1.

\section{Definition of recurrence}

Recurrences were identified by retrospective chart review in 389 patients. 21 of these had to be excluded due to incomplete survival data. Thus, a total of 368 patients were analyzed. Recurrence was arbitrarily defined as the first clinical manifestation of tumor progression after clinical remission following primary surgery and chemotherapy. Each recurrence was confirmed by radiologic imaging. In the majority of cases, cytological and/or the clinical course of disease confirmed tumor progression. In the remaining patients, serial imaging and/ or the clinical course of disease unequivocally confirmed tumor progression. Patients with an elevated CA-125 alone and those who had clinically evident tumor after primary surgery were not eligible for this study.

In the vast majority of patients, adjuvant chemotherapy consisted of cisplatinum-based regimens before 1990 and of carboplatin-based regimens thereafter. Carboplatin and paclitaxel combination therapy was used as a standard since the year 2000 . 
Table 1: Tumor and patient characteristics of the 368 evaluable consecutive patients with recurrent primary epithelial cancer of the ovary, fallopian tube and peritoneum following primary clinical remission.

\begin{tabular}{|c|c|}
\hline \multirow{2}{*}{ Characteristics } & Number of patients (\%) \\
\hline & $368(100 \%)$ \\
\hline Mean age; years (range) & $58(21-90)$ \\
\hline \multicolumn{2}{|l|}{ Age at primary diagnosis } \\
\hline$<70$ years & $287(78 \%)$ \\
\hline$\geq 70$ years & $81(22 \%)$ \\
\hline \multicolumn{2}{|l|}{ Tumor origin } \\
\hline Ovary & $344(93 \%)$ \\
\hline Fallopian tube & $14(4 \%)$ \\
\hline Peritoneum & $10(3 \%)$ \\
\hline \multicolumn{2}{|l|}{ FIGO stage } \\
\hline I-II & $65(18 \%)$ \\
\hline III-IV & $303(82 \%)$ \\
\hline \multicolumn{2}{|l|}{ Histology } \\
\hline Serous & $263(71 \%)$ \\
\hline Clear-cell & $40(11 \%)$ \\
\hline Endometrioid & $32(9 \%)$ \\
\hline Undifferentiated & $21(6 \%)$ \\
\hline Mucinous & $12(3 \%)$ \\
\hline \multicolumn{2}{|l|}{ Grading } \\
\hline G1 & $42(11 \%)$ \\
\hline $\mathrm{G} 2$ & $88(24 \%)$ \\
\hline G3 & $218(59 \%)$ \\
\hline Unknown & $20(6 \%)$ \\
\hline \multicolumn{2}{|l|}{ Ascites at the time of diagnosis } \\
\hline None & $130(35 \%)$ \\
\hline$<500 \mathrm{ml}$ & $78(21 \%)$ \\
\hline$>500 \mathrm{ml}$ & $160(44 \%)$ \\
\hline \multicolumn{2}{|l|}{ Type of primary surgery } \\
\hline $\mathrm{TAH}^{\mathrm{a}}+\mathrm{BSO} / \mathrm{USO}^{\mathrm{b}}+\mathrm{OM}+$ lymphadenectomy $^{\mathrm{c}}$ & $184(50 \%)$ \\
\hline $\mathrm{TAH}^{\mathrm{a}}+\mathrm{BSO} / \mathrm{USO}^{\mathrm{b}}+\mathrm{OM}^{\mathrm{d}}$ & $184(50 \%)$ \\
\hline \multicolumn{2}{|l|}{ Bowel resection at primary surgery } \\
\hline Yes & $110(30 \%)$ \\
\hline No & $256(69.5 \%)$ \\
\hline Unknown & $2(0.5 \%)$ \\
\hline \multicolumn{2}{|l|}{ Retroperitoneal lymph node status } \\
\hline Positive & $134(36 \%)$ \\
\hline Negative & $66(18 \%)$ \\
\hline Unknown & $168(46 \%)$ \\
\hline \multicolumn{2}{|l|}{ Residual disease at primary surgery } \\
\hline None & $158(43 \%)$ \\
\hline 0.1 to $1.0 \mathrm{~cm}$ & $45(12 \%)$ \\
\hline 1.1 to $1.9 \mathrm{~cm}$ & $59(16 \%)$ \\
\hline$\geq 2.0 \mathrm{~cm}$ & $106(29 \%)$ \\
\hline
\end{tabular}

\begin{tabular}{|c|c|}
\hline \multicolumn{2}{|l|}{ Adjuvant platinum-based chemotherapy } \\
\hline Yes & $316(86 \%)$ \\
\hline No & $52(14 \%)$ \\
\hline \multicolumn{2}{|l|}{ Age at first recurrence } \\
\hline$<70$ years & $300(82 \%)$ \\
\hline$\geq 70$ years & $57(15 \%)$ \\
\hline Unknown & $11(3 \%)$ \\
\hline \multicolumn{2}{|l|}{ Interval to first recurrence } \\
\hline$<12$ months & $132(36 \%)$ \\
\hline $12-24$ months & $173(47 \%)$ \\
\hline$>24$ months & $63(17 \%)$ \\
\hline \multicolumn{2}{|l|}{ Symptoms at recurrence } \\
\hline Yes & $(62 \%)$ \\
\hline No & $(5 \%)$ \\
\hline Unknown & $(32 \%)$ \\
\hline \multicolumn{2}{|c|}{ Karnofsky status at the time of recurrence/metastasis } \\
\hline$<80$ & $32(9 \%)$ \\
\hline$\geq 80$ & $336(91 \%)$ \\
\hline \multicolumn{2}{|l|}{$\mathrm{CA} 125>40 \mathrm{U} / \mathrm{ml}$ at the time of recurrence } \\
\hline$<200 \mathrm{U} / \mathrm{ml}$ & $49(13 \%)$ \\
\hline$\geq 200 \mathrm{U} / \mathrm{ml}$ & $47(13 \%)$ \\
\hline Unknown & $272(74 \%)$ \\
\hline \multicolumn{2}{|l|}{ Number of sites of recurrence } \\
\hline 1 & $140(38 \%)$ \\
\hline$\leq 2$ & $158(43 \%)$ \\
\hline$>5$ & $70(19 \%)$ \\
\hline \multicolumn{2}{|l|}{ Largest size of recurrent lesion/s } \\
\hline$<2 \mathrm{~cm}$ & $110(30 \%)$ \\
\hline$\geq 2 \mathrm{~cm}$ & $258(70 \%)$ \\
\hline \multicolumn{2}{|l|}{ Therapy for recurrence } \\
\hline Surgery and platinum-based chemotherapy & $14(4 \%)$ \\
\hline Platinum-based chemotherapy & $309(84 \%)$ \\
\hline Non-platinum-based chemotherapy & $26(7 \%)$ \\
\hline Palliative radiotherapy & $2(1 \%)$ \\
\hline Palliative supportive therapy only & $17(5 \%)$ \\
\hline
\end{tabular}

aTotal abdominal hysterectomy $(n=301)$; previous hysterectomy for benign disease $(n=67)$.

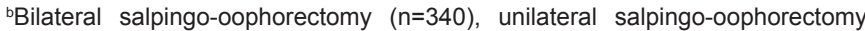
$(n=28)$.

'Pelvic $(n=184)$ paraaortic Lymphadenectomy $(n=70)$.

'Omentectomy $(n=368)$.

\section{Follow-up visits}

Follow-up examinations were scheduled every three months for three years after surgery and every six months until the fifth year after diagnosis. Follow-up had been standardized and carried out centrally at our institution for $95 \%$ of patients. Follow-up consisted of gynecologic clinical examinations every three months during the first three years as well as six-monthly CT scans thereafter until the end of the fifth year after diagnosis. 


\section{Predictive factors analyzed}

The following factors potentially affecting postrecurrence survival were analyzed:

- $\quad$ Primary tumor origin

- $\quad$ FIGO stage

- Histology

- $\quad$ Grading

- $\quad$ Age at primary diagnosis

- Ascites at the time of diagnosis

- $\quad$ Type of primary surgery

- Bowel resection at primary surgery

- $\quad$ Retroperitoneal lymph node status

- Residual disease at primary surgery

- $\quad$ Adjuvant platinum-based chemotherapy

- $\quad$ Age at first recurrence

- Interval to first recurrence

- Symptoms at recurrence

- Karnofsky status at the time of recurrence/metastasis

- $\quad \mathrm{CA} 125>40 \mathrm{U} / \mathrm{ml}$ at the time of recurrence

- $\quad$ Localization of recurrence

- Number of sites of recurrence

- $\quad$ Largest size of recurrent lesions

- Therapy for recurrence

\section{Statistical analysis}

Standard descriptives methods were used. Kaplan-Meier-curves were applied to describe patient survival from the time of recurrence, and Cox's proportional hazards model was used for multivariate analysis.

The analysis was done with IBM SPSS (IBM SPSS Statistics for Windows, V.22. Armonk, NY: IBM Corp).

\section{Results}

The mean follow-up of study patients was 17 months (range 0 to 146 months). The median post-recurrence survival was 11 months (range 0-146 months). Recurrences in the 368 patients were diagnosed after a median of 16 months (range 0-186 months). Sites of recurrence are shown in (Table 2).

Univariate analysis identified the following parameters to be significantly associated with favourable survival after first recurrence: FIGO stage I-II $(\mathrm{p}=0.000)$, tumor residuals of $\leq 1 \mathrm{~cm}$ in diameter at primary surgery $(\mathrm{p}=0.000)$, only one localization of recurrence $(\mathrm{p}=0.000)$, age $<70$ years at the time of recurrence $(\mathrm{p}=0.017)$, negative retroperitoneal lymph node status at primary surgery $(p=0.018)$, and peripheral lymph node recurrence $(\mathrm{p}=0.009)$, respectively.
Table 2: Localization of first recurrence/metastasis in the 368 evaluable consecutive patients with primary epithelial cancer of the ovary, fallopian tube and peritoneum.

\begin{tabular}{|c|c|}
\hline \multirow{2}{*}{ Characteristics } & Number of patients (\%) \\
\hline & $368(100 \%)$ \\
\hline Peritoneum & $90(25 \%)$ \\
\hline Peritoneum and pelvis & $14(4 \%)$ \\
\hline Pelvis only (no specification) & $87(24 \%)$ \\
\hline Pelvic side wall & $11(3 \%)$ \\
\hline Vaginal cuff & $15(4 \%)$ \\
\hline Middle and lower vagina & $4(1 \%)$ \\
\hline Liver & $22(6 \%)$ \\
\hline Liver and peritoneum & $15(4 \%)$ \\
\hline Liver and pelvis & $4(1 \%)$ \\
\hline Liver and pleura & $2(0.5 \%)$ \\
\hline Pleura & $8(2 \%)$ \\
\hline Pleura and peritoneum & $4(1 \%)$ \\
\hline Pleura, peritoneum and pelvis & $3(1 \%)$ \\
\hline Pleura and lung & $2(0.5 \%)$ \\
\hline Lung & $11(3 \%)$ \\
\hline Paraaortic lymph nodes only & $23(6 \%)$ \\
\hline Paraaortic lymph nodes and liver & $4(1 \%)$ \\
\hline Paraaortic and mediastinal lymph nodes & $1(0.3 \%)$ \\
\hline Paraaortic lymph nodes and peritoneum & $1(0.3 \%)$ \\
\hline Paraaortic lymph nodes and pelvis & $1(0.3 \%)$ \\
\hline Paraaortic lymph nodes, peritoneum and pelvis & $1(0.3 \%)$ \\
\hline Paraaortic lymph nodes and pleura & $1(0.3 \%)$ \\
\hline Supraclavicular lymph nodes & $9(2 \%)$ \\
\hline Supraclavicular lymph nodes and pelvis & $1(0.3 \%)$ \\
\hline Inguinal lymph nodes & $7(2 \%)$ \\
\hline Inguinal lymph nodes and peritoneum & $1(0.3 \%)$ \\
\hline \multicolumn{2}{|l|}{ Inguinal and axillary lymph nodes, } \\
\hline peritoneum and lung & $1(0.3 \%)$ \\
\hline Brain & $8(2 \%)$ \\
\hline Spleen & $4(1 \%)$ \\
\hline Spleen and peritoneum & $3(1 \%)$ \\
\hline Skin & $4(1 \%)$ \\
\hline Umbilicus & $3(1 \%)$ \\
\hline Abdominal wall & $3(1 \%)$ \\
\hline
\end{tabular}

In multivariate Cox regression analysis, five parameters were identified as independent favorable predictive factors for survival after first recurrence: Time to recurrence $\geq 2$ years ( $p=0.000$; Figure 1 ), Karnofsky status $\geq 80 \%$ at the time of recurrence ( $p=0.008$, Figure 2), use of adjuvant platinum-based chemotherapy ( $\mathrm{p}=0.013)$, residual disease $\leq 1 \mathrm{~cm}$ at primary surgery ( $\mathrm{p}=0.044$, Figure 3 ) and isolated peripheral or paraaortic lymph nodes as localization of first recurrence ( $\mathrm{p}<0.05$; Figure 4$)$, respectively. 


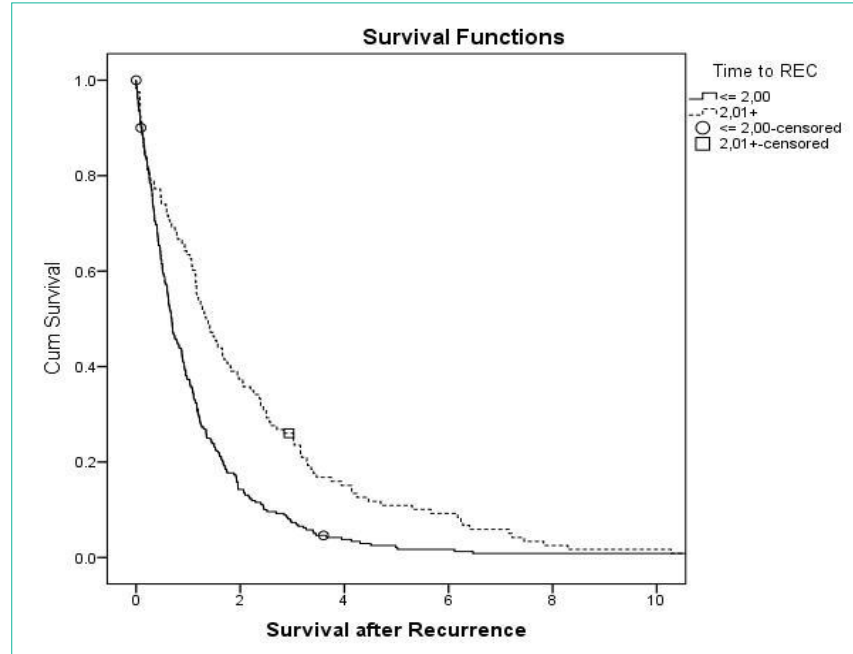

Figure 1: Overall cumulative survival of patients with primary epithelial cancer of the ovary, fallopian tube, or peritoneum whose interval between primary diagnosis and recurrence was less than or equal two years versus those with an interval of greater than two years.

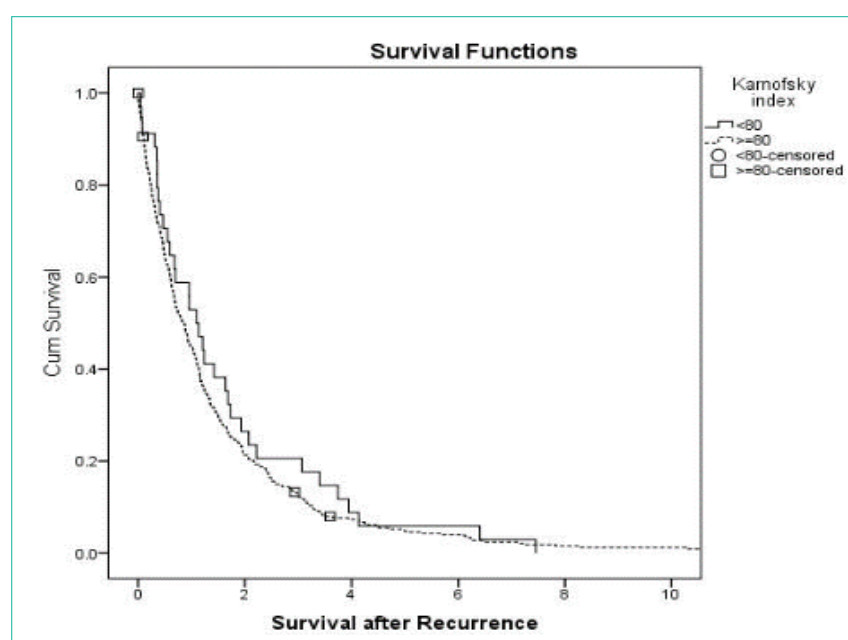

Figure 2: Overall cumulative survival of patients with primary epithelial cancer of the ovary, fallopian tube, or peritoneum who either had a Karnofsky status of $<80 \%$ or $\geq 80 \%$ at the time of recurrence.

\section{Discussion}

In 368 patients who developed recurrence after clinical remission, postrecurrence survival was favorably influenced by an interval of $\geq$ 2 years from primary surgery (Figure 1), small or no residual disease at primary surgery (Figure 3), the use of adjuvant platinum-based chemotherapy, a higher Karnofsky status at recurrence (Figure 2) and recurrence/metastases in the peripheral or paraaortic lymph nodes (Figure 4).

There are only three studies including higher patient numbers with ovarian cancer recurrence $[9,10,13]$. However, one of these had focussed on the response of patients to chemotherapy only [13].

\section{Longer interval to recurrence}

Our study identified a disease-free interval of two years from diagnosis as predictor of improved survival (Figure $1 ; \mathrm{p}=0.000$ ). This finding supports several other reports $[3-8,10,11]$.

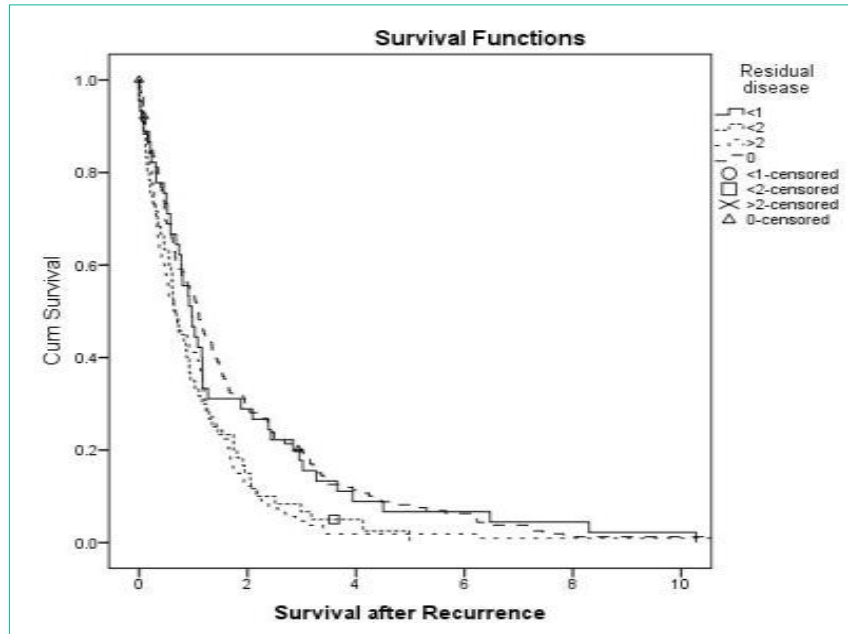

Figure 3: Overall cumulative survival of patients with primary epithelial cancer of the ovary, fallopian tube, or peritoneum who either had no residual disease, residual disease $<1 \mathrm{~cm}$, residual disease between 1.0 and $1.9 \mathrm{~cm}$, or $\geq 2 \mathrm{~cm}$ in largest diameter at primary debulking surgery.

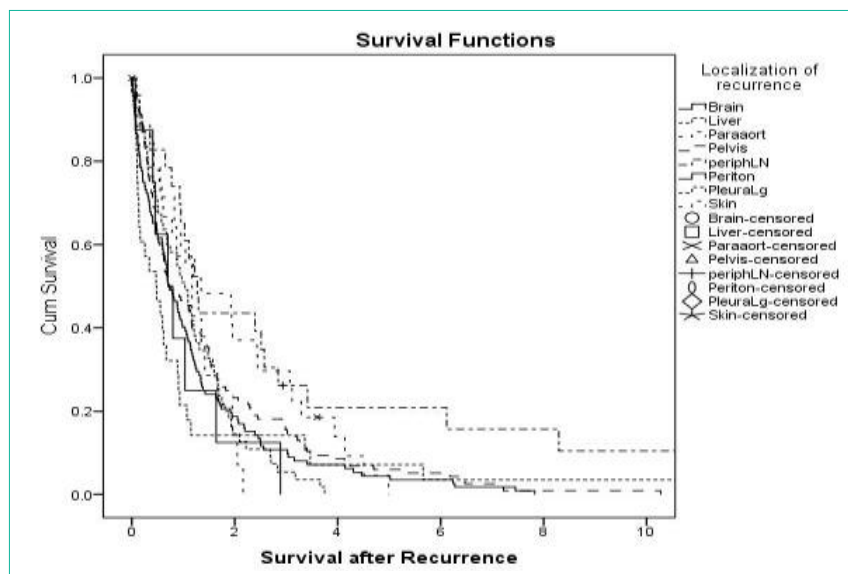

Figure 4: Overall cumulative survival of patients with primary epithelial cancer of the ovary, fallopian tube, or peritoneum who either developed their first recurrence in the brain, parenchymatous liver, paraaortic lymph nodes, pelvis, peripheral lymph nodes, peritoneum, pleura and lungs, and skin, respectively.

\section{Residual disease $\leq 1 \mathrm{~cm}$ at primary surgery}

In the present study, minimal or no residual disease at primary surgery was an independent favorable factor for postrecurrence survival (Figure 3; $\mathrm{p}=0.044$ ). This finding is supported by another report on pooled data from 3 randomized AGO studies from the German Working Group for Gynecologic Oncology [9].

\section{Adjuvant chemotherapy}

The administration of adjuvant platinum-based chemotherapy was identified as favourable predictive factor for prognosis in the present series $(\mathrm{p}=0.013)$. We were unable to identify other studies showing a similar finding.

\section{Higher performance status at the time of recurrence}

Our findings revealed a Karnofsky status $\geq 80 \%$ at the time of recurrence to be predictive for postrecurrence survival $(\mathrm{p}=0.008$; Figure 2). Other groups have shown similar results $[3,6]$. 


\section{Localization of recurrence}

The most common first clinical manifestations of recurrent ovarian cancer were metastases of the peritoneal cavity, followed by the pelvis. Isolated lymph node recurrences, mainly concerning the paraaortic area, were less common (Table 2).

\section{Peritoneal metastases}

It seems evident that the term peritoneal cavity was not uniformly used in the studies reported since some authors have not distinguished between recurrences restricted to the pelvis and those involving both the pelvis and the peritoneal cavity [1,14-16]. The present study did not identify a prognostic difference between these two recurrence patterns (Figure 4).

Peritoneal metastases have been identified as a component of first recurrence in $36 \%$ of our patients (Table 2). This data are in line with those from another study (38\% peritoneal recurrences; [1]). Amate et al. reported peritoneal involvement as site of first recurrence in $77 \%$ of patients, most of them having advanced disease [16].

In autopsy studies, up to $96 \%$ of patients were identified to have peritoneal metastatic involvement $[14,15,17]$.

\section{Pelvic metastases}

In our cohort, $34 \%$ of patients showed first recurrences in the pelvis (Table 2). In contrast, an autopsy study revealed even a higher rate of pelvic peritoneal metastases (65\%; [17]).

\section{Isolated vaginal cuff recurrences}

In our collective, 15 patients (4\%) developed an isolated recurrence of the vaginal cuff (Table 2). This is in accordance with another study in which $5 \%$ such recurrences were reported [18].

\section{Liver metastases}

Parenchymatous liver metastases occurred in $13 \%$ of our patients (Table 2). Dauplat et al. reported 14\% liver metastases [19]. Autopsy studies in patients with ovarian cancer have observed liver metastases between 45 and $52 \%$ of patients [14,15,17].

\section{Peripheral and paraaortic lymph node recurrences}

In the present series, metastatic involvement of peripheral or paraaortic lymph nodes was found in 51 cases 39 of which (77\%) were isolated (Table 2). Overall, peripheral and paraaortic lymph node recurrences represented $11 \%$ of recurrences in this series. Paraaortic recurrences were treated by secondary surgery in 10 patients. Peripheral or paraaortic lymph node involvement was a favorable predictor of survival in the present study (Figure $4 ; \mathrm{p}<0.05$ ). This data is in line with several previous reports in which survival up to $>100$ months was recorded [11,20-22].

\section{Pleural metastases}

About $5 \%$ of our patients had pleural metastases as primary localization of recurrence (Table 2). In studies with far advanced disease and autopsy studies, the proportion of pleural involvement lay between 28 and 59\%, respectively [14,15,17]. In a report on 97 patients first recurring at distant sites, 59\% had malignant pleural effusions [19]. Another study showed thoracic involvement in $45 \%$ of patients the vast majority of them having pleural effusions [23].

\section{Lung metastases}

In the present study, parenchymal lung metastases were found as primary manifestation of recurrence in $4 \%$ of patients only (Table 2). This number corresponds well to one other study [23]. Autopsy studies have found a prevalence of lung metastases in up to $39 \%$ $[14,15,17]$.

\section{Spleen metastases}

Two percent of patients in the present series showed metastatic involvement of the spleen (Table 2). Previous studies have revealed rates between $4 \%$ and $10 \%$, respectively $[1,2]$. However, autopsy studies have reported spleen metastases in $19 \%$ to $51 \%$ of patients, respectively $[14,15,17]$.

\section{Brain/CNS metastases}

In the present cohort, $2 \%$ of patients were diagnosed with brain metastases (Table 2). These data are in line with previous observations by Dauplat et al [19]. Autopsy studies have revealed up to $6 \%$ brain metastases from ovarian cancer $[14,15,17]$.

\section{Skin metastases}

In the present series, four patients (1\%) developed skin metastases (Table 2). These data are in line with Cormio et al. who described $4 \%$ skin metastases among 220 patients with epithelial ovarian cancer [2]. Autopsy studies revealed prevalence rates of as high as 5\% [15,17].

\section{Number of sites of recurrence}

The number of sites of recurrence was not predictive for postrecurrence survival in the present series. However, various and inconsistent methods have been applied in order to diagnose the localization of recurrences/metastases. For example, a patient might have undergone emergency surgery for an ileus at the time of first recurrence at which numerous metastases were stated. In another case, a patient with significant dyspnea might have been diagnosed via chest X-ray only without performing further imaging. In the present study, this patient might have been classified as having one localization of recurrence only despite the coexistence of several other metastatic lesions identified 2 weeks later by thoracoscopy.

In one study, the number of disease sites at recurrence was significantly associated with response. However, the authors did not report on overall survival [13]. Biliatis et al. reported that three or more sites of recurrence are independently associated with poor prognosis [7]. Pignata et al. reported similar effects on overall survival [6].

\section{Maximum tumor size at recurrence}

This parameter was not significantly associated with survival in the present dataset. One pooled study found tumor size of recurrence $\leq 5 \mathrm{~cm}$ to be independently associated with improved response [13].

\section{FIGO stage}

In the present cohort, 303 patients (82\%) had FIGO stage III or IV disease. Stage had no influence on postrecurrence survival. In contrast, two other studies have identified advanced stage of disease as a negative independent prognostic factor post recurrence $[7,10]$.

\section{Histological subtype}

The histological subtype had no predictive value in the present 
series. Only singular studies have found serous histology to be significantly associated with an improved response and prognosis $[7,10,13]$.

\section{CA 125}

In the present study, CA-125 values were available only in $26 \%$ of patients overall (Table 1). No predictive value was found. Another group also reported no prognostic significance for preoperative CA 125 evaluated in patients before secondary cytoreduction [5].

\section{Other factors investigated}

No difference in postrecurrence survival was found with regard to the origin of the primary tumor, grading, age at diagnosis and recurrence, the presence of ascites at primary surgery, whether primary surgery included lymphadenectomy or not, retroperitoneal lymph node status, and type of treatment of recurrence. However, it has to be stated that only a minority of our patients underwent secondary debulking surgery (4\%; Table 1$)$. There are several papers in the literature which have shown secondary debulking surgery at the time of recurrence to positively influence survival in selected patients if residual disease is low [24].

\section{Conclusion}

In this series, the time to recurrence was the strongest predictor of postrecurrence survival. In addition, small or no residual tumor after primary surgery, the administration of adjuvant chemotherapy, a higher performance status at recurrence, and metastases in the peripheral or paraaortic nodes are predictive for overall survival. Thus, even parameters relevant at the time of diagnosis and shortly thereafter, such as residual tumor size and/or adjuvant chemotherapy influence postrecurrence survival of patients with recurrent ovarian cancer.

\section{Author Contributions}

All authors have contributed intellectually to the manuscript.

EP: Study concept and design, acquisition of clinical data, the use of manuscript writing.

\section{TI: Acquisition of clinical data, manuscript writing.}

NW: Acquisition of clinical data, manuscript writing.

$\mathrm{JH}$ : Statistical analysis, interpretation of data.

$\mathrm{CB}$ : Acquisition of clinical data, manuscript writing.

$\mathrm{MH}$ : Acquisition of clinical data, manuscript writing.

SK: Acquisition of clinical data, manuscript writing.

KW: Acquisition of clinical data, manuscript writing.

KT: Manuscript editing.

\section{References}

1. Kikkawa F, Kawai M, Mizuno K, Ishikawa H, Kojima M, Maeda O, et al Recurrence of epithelial ovarian carcinoma after clinical remission. Gynecol Obstet Invest. 1994; 38: 65-69.

2. Cormio G, Rossi C, Cazzolla A, Resta L, Loverro G, Greco P, et al. Distant metastases in ovarian carcinoma. Int J Gynecol Cancer. 2003; 13: 125-129.

3. Begum F, Høgdall E, Riisbro R, Christensen I, Engelholm S, Jørgensen M, et al. Prognostic value of plasma soluble urokinase Plasminogen Activator
Receptor (suPAR) in Danish patients with Recurrent Epithelial Ovarian Cancer (REOC). APMIS. 2006; 114: 675-681.

4. Chan JK, Tian C, Teoh D, Monk BJ, Herzog T, Kapp DS, et al. Survival after recurrence in early-stage high-risk epithelial ovarian cancer: a Gynecologic Oncology Group study. Gynecol Oncol. 2010; 116: 307-311.

5. Mahner S, Woelber L, Jung S, Eulenburg C, Ihnen M, Schwarz J, et al. Prognostic significance of CA-125 in the management of patients with recurrent epithelial ovarian carcinoma selected for secondary cytoreduction. Anticancer Res. 2009; 29: 2817-2821.

6. Pignata S, Ferrandina G, Scarfone G, Scollo P, Odicino F, Cormio G, et al. Poor outcome of elderly patients with platinum-sensitive recurrent ovarian cancer: results from the SOCRATES retrospective study. Crit Rev Oncol Hematol. 2009; 71: 233-241.

7. Biliatis I, Haidopoulos D, Rodolakis A, Vlachos G, Protopapas A, Thomakos $\mathrm{N}$, et al. Survival after secondary cytoreduction for recurrent ovarian cancer: which are the prognostic factors?. J Surg Oncol. 2010; 102: 671-675.

8. Classe J, Jaffre I, Frenel J, Bordes V, Dejode M, Dravet F, et al. Prognostic factors for patients treated for a recurrent FIGO stage III ovarian cancer: a retrospective study of 108 cases. Eur J Surg Oncol. 2011; 37: 971-977.

9. Hanker L, Loibl S, Burchardi N, Pfisterer J, Meier W, Pujade-Lauraine E, et al. The impact of second to sixth line therapy on survival of relapsed ovarian cancer after primary taxane/platinum-based therapy. Ann Oncol. 2012; 23: 2605-2612.

10. Kajiyama H, Shibata K, Mizuno M, Umezu T, Suzuki S, Yamamoto E, et al. Long-term clinical outcome of patients with recurrent epithelial ovarian carcinoma: is it the same for each histological type?. Int J Gynecol Cancer. 2012; 22: 394-399.

11. Jayson GC, Kohn EC, Kitchener HC, Ledermann JA. Ovarian cancer. Lancet. 2014; 384: 1376-1388.

12. Gadducci A, Cosio S, Zola P, Sostegni B, Fuso L, Sartori E. Prognostic factors and clinical outcome of patients with recurrent early-stage epithelial ovarian cancer: an Italian multicenter retrospective study. Int J Gynecol Cancer. 2013; 23: 461-468.

13. Eisenhauer E, Vermorken J, van Glabbeke M. Predictors of response to subsequent chemotherapy in platinum pretreated ovarian cancer: a multivariate analysis of 704 patients. Ann Oncol. 1997; 8: 963-968.

14. Abrams H, Spiro R, Goldstein N. Metastases in carcinoma; analysis of 1000 autopsied cases. Cancer. 1950; 3: 74-85.

15. Rose P, Piver M, Tsukada Y, Lau T. Metastatic patterns in histologic variants of ovarian cancer. An autopsy study. Cancer. 1989; 64: 1508-1513.

16. Amate $P$, Huchon $C$, Dessapt $A$, Bensaid $C$, Medioni J, Le Frère Belda $M$, et al. Ovarian cancer: sites of recurrence. Int J Gynecol Cancer. 2013; 23: 1590-1596.

17. Dvoretsky P, Richards K, Angel C, Rabinowitz L, Stoler M, Beecham J, et al. Distribution of disease at autopsy in 100 women with ovarian cancer. Hum Pathol. 1988; 19: 57-63.

18. Casey A, Park M, Holschneider C, Bozuk M, Punyasavatsut M, Montz F. Apical vaginal recurrence of ovarian carcinoma: presentation, treatment and survival. Int J Gynecol Cancer. 1996; 6: 200-204.

19. Dauplat J, Hacker N, Nieberg R, Berek J, Rose T, Sagae S. Distant metastases in epithelial ovarian carcinoma. Cancer. 1987; 60: 1561-1566.

20. Uzan C, Morice P, Rey A, Pautier P, Camatte S, Lhomme C, et al. Outcomes after combined therapy including surgical resection in patients with epithelial ovarian cancer recurrence(s) exclusively in lymph nodes. Int J Gynecol Cancer (Abstracts). 2004; 14: 42-43.

21. Blanchard $P$, Plantade $A$, Pagès $C$, Afchain $P$, Louvet $C$, Tournigand $C$, et al. Isolated lymph node relapse of epithelial ovarian carcinoma: outcomes and prognostic factors. Gynecol Oncol. 2007; 104: 41-45.

22. Legge F, Petrillo M, Adamo V, Pisconti S, Scambia G, Ferrandina G. Epithelial 
ovarian cancer relapsing as isolated lymph node disease: natural history and clinical outcome. BMC Cancer. 2008; 8: 367

23. Kerr V, Cadman E. Pulmonary metastases in ovarian cancer. Cancer. 1985. 56: 1209-1213.
24. Harter P, Sehouli J, Reuss A, Hasenburg A, Scambia G, Cibula D, et al. Prospective validation study of a predictive score for operability of recurrent ovarian cancer: the Multicenter Intergroup Study DESKTOP II. A project of the AGO Kommission OVAR, AGO Study Group, NOGGO, AGO-Austria, and MITO. Int J Gynecol Cancer. 2011; 21: 289-295.
Ann Hematol Oncol - Volume 5 Issue 8 - 2018

ISSN : 2375-7965 | www.austinpublishinggroup.com

Petru et al. (C) All rights are reserved
Citation: Petru E, Idris T, Woltsche N, Haas J, Benedicic C, Heydar-Fadai M, et al. Predictive Factors for Postrecurrence Survival in Epithelial Ovarian Cancer-a Consecutive Series of 368 Patients and Review of the Literature. Ann Hematol Oncol. 2018; 5(8): 1225 